\title{
New report of Penicillium implicatum causing a postharvest rot of pomegranate fruit in Pakistan
}

\author{
Ibatsam Khokhar • Rukhsana Bajwa • Ghazala Nasim
}

Received: 12 September 2011 / Accepted: 18 January 2013/Published online: 31 January 2013

(C) Australasian Plant Pathology Society Inc. 2013

\begin{abstract}
In this study Penicillium implicatum was found to be the cause of postharvest rot of stored pomegranate (Punica granatum $L$.) fruit. Rot symptom was observed on pomegranate fruit as small, sunken, circular to oval, dark brown necrotic spots. Infected fruit tissues were cultured on malt extract agar (MEA), Czapek (Cz), Czapek yeast Agar (CYA) and G25N media at $25^{\circ} \mathrm{C}$. This fungal species was primarily characterized by its relatively slow growth on MEA, Cz, CYA and $\mathrm{G} 25 \mathrm{~N}$, blue-grey sporulation, forming crusts, production of a soluble yellow pigment on the media and inability to grow at 5 and $37^{\circ} \mathrm{C}$ on both $\mathrm{Cz}$ and MEA. Microscopically, it was characterized by its long, smooth-walled and vesiculate conidiophores and smooth ellipsoidal to ovoid, up to $3.5 \mu \mathrm{m}$ long, conidia. The fungus was identified as P. implicatum on the basis of morphological and cultural characteristics. Pathogenicity tests conducted on healthy fruits under laboratory conditions showed typical rot symptoms after 7 to 14 days. This is the first report of postharvest rot of pomegranate caused by P. implicatum in Pakistan.
\end{abstract}

\section{Keywords Punica granatum · Penicillium implicatum . Fruit rot $\cdot$ Pomegranate}

Penicillium is one of the major causes of spoilage of agricultural products during pre and post harvest phase. In December 2010, during a survey of local fruit market in Lahore (Pakistan), irregular brown to caramel-brown spots were observed on the skin of pomegranate fruits (Punica

I. Khokhar $(\bowtie) \cdot$ R. Bajwa $\cdot$ G. Nasim

Institute of Agricultural Sciences, University of the Punjab,

Lahore, Pakistan

e-mail: ibatsamk@yahoo.com granatum L.). To clarify the causal agents of those symptoms, fruit samples were obtained from a local fruit market, kotlakhpat, Lahore and examined at laboratory. From the necrotic areas, a blue-grey fungal growth was observed. Temporary slides of diseased tissues were made and observed under light microscope. Small pieces $(3 \mathrm{~mm}$ ) of rotting tissue from the fruits and surface sterilized with $1 \% \mathrm{Na}(\mathrm{O}) \mathrm{Cl}$, were placed onto $2 \%$ malt extract agar (MEA) and incubated at $25^{\circ}$ $\mathrm{C}$ in darkness for 5 days. As a result, a species belonging to the genus Penicillium subgenus Aspergilloides was consistently found associated to the described symptoms. A recent record proves that P. implicatum Biourge (subgenus Aspergilloides) was the causal agent of a similar fruit rot in Slovak Republic (Labuda et al. 2004). According to key (Raper and Thom 1949; Pitt 1979, 1985; Ramírez 1982; Samson et al. 1995; Pitt and Hocking 1997) this species is primarily characterized by its relatively slow growth on Czapek-based media and MEA at $25^{\circ} \mathrm{C}$, heavy blue-grey sporulation, forming crusts, production of a soluble yellow pigment on the media, and inability to grow at 5 and $37^{\circ} \mathrm{C}$ on both $\mathrm{Cz}$ and MEA. Thus, in order to allow the confirmation of the fungus identity obtained in this study the resulting fungal colonies were subcultured on Czapek-solution agar (Cz), $2 \%$ MEA, CYA and G25N at $25^{\circ}$ C for 7 to 14 days according to Pitt (1979).

The description of our fungal specimen is as follow: On $\mathrm{Cz}$ medium, colonies 20-25 mm diam., surface velutinous, somewhat floccose in the centres, plane, centrally raised or crateriform, margins arachnoid; mycelium white, occasionally conspicuous in the centers; dense colonies with profuse sporulation showing some tendency to form crusts in age, bluish grey; exudate absent, yellow or dull reddish-brown soluble pigment produced, reverse yellowish to reddish brown or maroon in the presence of soluble pigment. On MEA medium, colonies 20-25 mm diam., velutinous, plane, 

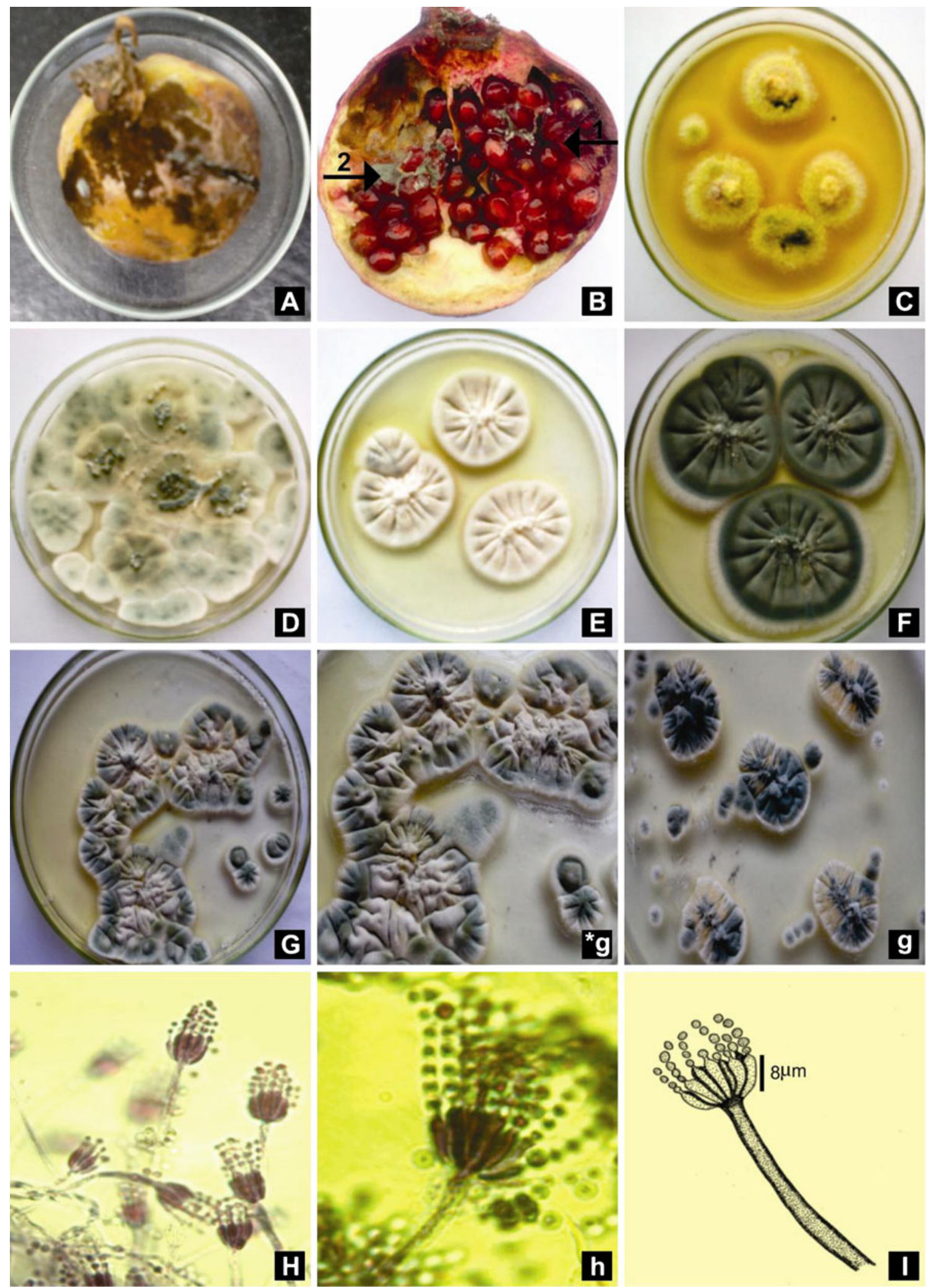

Fig. 1 Postharvest infections of Punica granatum L. by Penicillium implicatum. a Infected Pomegranate b Postharvest infection of P.implicatum (1) necrosis of the tissue (2) fungal growth (C-g) P. implicatum growth on MEA, Czapek-solution agar, CYA and G25N, respectively. (H) Hyphae and penicillius (h) Shape of penicillius (I) Conidiophore producing conidia 
margins entire; mycelium white, inconspicuous; dense colonies with profuse sporulation with tendency to form crusts, grayish blue; no exudates; yellow soluble pigment mostly produced, reverse pale or in bright yellow shades, yellowish in the presence of soluble pigment. On CYA medium colonies were variable in size from $15 \mathrm{~mm}$ to $25 \mathrm{~mm}$ in diameter, slow growing, radially sulcate and centrally umbonate, velutinous, mycelium white, cream or buff. Mycelium became grayish green or dull green due to conidiogenesis. Reverse of the colony was yellow brown. Exudates and brown soluble pigment present. On G25N medium colonies were variable in size from $0.5 \mathrm{~mm}$ to $10 \mathrm{~mm}$ in diameter, slow growing, umbonate, velutinous, ocassionally sulcate. Mycelium became grayish green or dull green due to moderate conidiogenesis. Reverse of the colony was pale, olive or brown. Exudates and brown soluble pigment present (Fig. 1).

Microscopic examinations were conducted by mounting small portion of fungal colony in water and trypan blue, and dimensions of conidia were measured from seven day-old cultures. Conidiophores borne mostly from substrate, stipes (70-) 100-150 $(-250) \times 2.5-3.0 \mu \mathrm{m}$, thin walled, smooth; penicilli strictly monoverticillate, vesiculate $4.9-6.0 \mu \mathrm{m}$ width; phialides flask shaped, 8 to 20 in the verticil, with $7.5-8.5(-10) \times 2.4-2.9 \mu \mathrm{m}$; conidia ellipsoidal to ovoid (2.5-) 3.0-3.2 (-3.5) ×2.0-2.3 $\mu \mathrm{m}$, smooth walled.

Pathogenicity of the isolated organism was confirmed on healthy pomegranate fruits. Conidial suspension $\left(2 \times 10^{4}\right.$ conidia $\mathrm{mL}^{-1}$ ) from a pure culture of the fungus was directly inoculated by means of a sterile needle into the subcutaneous layer of fruits of pomegranate. Infested fruits were incubated at $25^{\circ} \mathrm{C}$ for 7 to 14 days. Typical symptoms were produced on the inoculated fruits after 7 days. The pathogen from the inoculated fruits was re-isolated on $2 \%$ MEA medium as described above. The morphological and cultural characteristics of the re-isolated organism were compared with the original pathogen. The pathogen was identified from all infected fruit samples. In a survey of pomegranate (Punica granatum) postharvest losses in commercial markets in Lahore, Pakistan, blue mould symptoms were observed on up to $20 \%$ and $10 \%$ of pomegranates.

Based on symptoms, pathogenicity on pomegranate fruit, and its morphological and cultural characteristics, as described above, the fungus was identified as being Penicillium implicatum Biourge. According to the literature, this is the first report of a postharvest rot of Pomegranate caused by $P$. implicatum in Pakistan and only second one to the same reported at international level by Labuda et al. 2004. A culture of the fungus has also been deposited at First fungal culture bank of Pakistan (FCBP), Institute of Agricultural Sciences, University of the Punjab Lahore, Pakistan, for further studies.

\section{References}

Labuda R, Hudec K, Piecková E, Mezey J, Bohovič R, Mátéová S, Lukáč SS (2004) Penicillium implicatum causes a destructive rot of pomegranate fruits. Mycopathologia 157:217-223

Pitt JI (1979) The genus Penicillium and its teleomorphic states Eupenicillium and Talaromyces. Academic, London

Pitt JI (1985) A laboratory guide to common Penicillium species. Commonwealth Scientific and Industrial Research Organization Division of Food Research, North Ryde

Pitt JI, Hocking AD (1997) Fungi and food spoilage. Academic Press, Chapman and Hall, Sydney

Ramírez C (1982) Manual and atlas of the Penicillia. Elsevier Biomedical Press, Amsterdam/New York/Oxford

Raper KB, Thom CH (1949) A manual of the Penicillia. Williams and Wilkins, Baltimore

Samson RA, Hoekstra ES, Frisvad JC, Filtenborg O (1995) Introduction to food-borne fungi. Centraalbureau voor Schimmelcultures, Baarn 